\title{
Complete monotonicity of a function involving the $p$-psi function and alternative proofs
}

\author{
Valmir Krasniqi ${ }^{1} \&$ Feng $\mathbf{Q i}^{2,3,4, *}$ \\ ${ }^{1}$ Department of Mathematics, University of Prishtina, Prishtinë 10000, Republic of Kosova \\ ${ }^{2}$ College of Mathematics, Inner Mongolia University for Nationalities, \\ Tongliao City, Inner Mongolia Autonomous Region, 028043, China \\ ${ }^{3}$ Department of Mathematics, College of Science, Tianjin Polytechnic University, Tianjin City, 300387, China \\ ${ }^{4}$ Institute of Mathematics, Henan Polytechnic University, Jiaozuo City, Henan Province, 454010, China \\ *Corresponding author's e-mail: qifeng618@gmail.com,qifeng618@hotmail.com,qifeng618@qq.com \\ *Corresponding author's URL: http: // qifeng618. wordpress. com
}

Copyright (C)2014 Valmir Krasniqi\& Feng Qi. This is an open access article distributed under the Creative Commons Attribution License, which permits unrestricted use, distribution, and reproduction in any medium, provided the original work is properly cited.

\begin{abstract}
In the paper, the authors prove that the function $x^{\alpha}\left[\ln \frac{p x}{x+p+1}-\psi_{p}(x)\right]$ is completely monotonic on $(0, \infty)$ if and only if $\alpha \leq 1$, where $p \in \mathbb{N}$ and $\psi_{p}(x)$ is the $p$-analogue of the classical psi function $\psi(x)$.
\end{abstract}

Keywords: completely monotonic function; necessary and sufficient condition; p-gamma function; p-psi function; inequality MSC: Primary 33D05; Secondary 26A48, 33B15, 33E50

\section{Introduction}

Recall from [12, Chapter XIII], [16, Chapter 1] and [17, Chapter IV] that a function $f$ is said to be completely monotonic on an interval $I$ if $f$ has derivatives of all orders on $I$ and satisfies

$0 \leq(-1)^{n} f^{(n)}(x)<\infty$

for $x \in I$ and $n \geq 0$. The celebrated Bernstein-Widder's Theorem (see [16, p. 3, Theorem 1.4] or [17, p. 161, Theorem 12b]) characterizes that a necessary and sufficient condition that $f(x)$ should be completely monotonic for $0<x<\infty$ is that

$f(x)=\int_{0}^{\infty} e^{-x t} \mathrm{~d} \alpha(t)$,

where $\alpha(t)$ is non-decreasing and the integral converges for $0<x<\infty$. This expresses that a completely monotonic function $f$ on $[0, \infty)$ is a Laplace transform of the measure $\alpha$. 
It is common knowledge that the classical Euler's gamma function $\Gamma(x)$ may be defined for $x>0$ by

$\Gamma(x)=\int_{0}^{\infty} t^{x-1} e^{-t} \mathrm{~d} t$.

The logarithmic derivative of $\Gamma(x)$, denoted by $\psi(x)=\frac{\Gamma^{\prime}(x)}{\Gamma(x)}$, is called psi function or digamma function.

An alternative definition of the gamma function $\Gamma(x)$ is

$\Gamma(x)=\lim _{p \rightarrow \infty} \Gamma_{p}(x)$,

where

$\Gamma_{p}(x)=\frac{p ! p^{x}}{x(x+1) \cdots(x+p)}=\frac{p^{x}}{x(1+x / 1) \cdots(1+x / p)}$

for $x>0$ and $p \in \mathbb{N}$, the set of all positive integers. See [3, p. 250]. The $p$-analogue of the psi function $\psi(x)$ is defined as the logarithmic derivative of the $\Gamma_{p}$ function, that is,

$\psi_{p}(x)=\frac{\mathrm{d}}{\mathrm{d} x} \ln \Gamma_{p}(x)=\frac{\Gamma_{p}^{\prime}(x)}{\Gamma_{p}(x)}$.

The function $\psi_{p}$ has the following properties:

1. It has the following representations

$$
\psi_{p}(x)=\ln p-\sum_{k=0}^{p} \frac{1}{x+k}=\ln p-\int_{0}^{\infty} \frac{1-e^{-(p+1) t}}{1-e^{-t}} e^{-x t} \mathrm{~d} t .
$$

2. It is increasing on $(0, \infty)$ and $\psi_{p}^{\prime}$ is completely monotonic on $(0, \infty)$.

The very right hand side of the formula (1.6) corrects errors appeared in [8, p. 374, Lemma 5] and [10, p. 29, Lemma 2.3].

In [2, pp. 374-375, Theorem 1], it was proved that the function

$\theta_{\alpha}(x)=x^{\alpha}[\ln x-\psi(x)]$

is completely monotonic on $(0, \infty)$ if and only if $\alpha \leq 1$. For the history, background, applications and alternative proofs of this conclusion, please refer to [4], [13, p. 8, Section 1.6.6] and closely related references therein.

The aim of this paper is to generalize [2, pp. 374-375, Theorem 1] and [4, p. 105, Theorem 1] to the case of the $p$-analogue $\psi_{p}(x)$ of the psi function $\psi(x)$ as follows.

Theorem 1.1. The function

$\theta_{p, \alpha}(x)=x^{\alpha}\left[\ln \frac{p x}{x+p+1}-\psi_{p}(x)\right]$

for $p \in \mathbb{N}$ is completely monotonic on $(0, \infty)$ if and only if $\alpha \leq 1$.

Remark 1.1. Letting $p \rightarrow \infty$ in Theorem 1.1, we obtain [2, pp. 374-375, Theorem 1] and [4, p. 105, Theorem 1].

\section{Proofs of Theorem 1.1}

First Proof. From the identity (1.6) and the integral expression

$\ln \frac{b}{a}=\int_{0}^{\infty} \frac{e^{-a t}-e^{-b t}}{t} \mathrm{~d} t$

in $[1$, p. $230,5.1 .32]$, we obtain

$\theta_{p, 1}(x)=x \int_{0}^{\infty}\left[1-e^{-(p+1) t}\right] \varphi(t) e^{-x t} \mathrm{~d} t$, 
where

$\varphi(t)=\frac{1}{1-e^{-t}}-\frac{1}{t}$.

The function $\varphi(t)$ is increasing on $(0, \infty)$ with

$\lim _{t \rightarrow 0^{+}} \varphi(t)=\frac{1}{2} \quad$ and $\quad \lim _{t \rightarrow \infty} \varphi(t)=1$.

See $[5,6,7,11,14,15,18]$ and related references therein. Therefore, for $x>0$ and $n \in \mathbb{N}$, we have

$$
\begin{aligned}
(-1)^{n} \theta_{p, 1}^{(n)}(x) & =x(-1)^{n} \frac{\mathrm{d}^{n}}{\mathrm{~d} x^{n}} \int_{0}^{\infty}\left[1-e^{-(p+1) t}\right] \varphi(t) e^{-x t} \mathrm{~d} t-(-1)^{n-1} n \frac{\mathrm{d}^{n-1}}{\mathrm{~d} x^{n-1}} \int_{0}^{\infty}\left[1-e^{-(p+1) t}\right] \varphi(t) e^{-x t} \mathrm{~d} t \\
& =x \int_{0}^{\infty} t^{n} \varphi(t)\left[1-e^{-(p+1) t}\right] e^{-x t} \mathrm{~d} t-n \int_{0}^{\infty} t^{n-1} \varphi(t)\left[1-e^{-(p+1) t}\right] e^{-x t} \mathrm{~d} t \\
& =\int_{0}^{n / x} t^{n-1}\left[1-e^{-(p+1) t}\right] \varphi(t)(t x-n) e^{-x t} \mathrm{~d} t+\int_{n / x}^{\infty} t^{n-1}\left[1-e^{-(p+1) t}\right] \varphi(t)(t x-n) e^{-x t} \mathrm{~d} t \\
& >\varphi\left(\frac{n}{x}\right) \int_{0}^{n / x} t^{n-1}\left[1-e^{-(p+1) t}\right](t x-n) e^{-x t} \mathrm{~d} t+\varphi\left(\frac{n}{x}\right) \int_{n / x}^{\infty} t^{n-1}\left[1-e^{-(p+1) t}\right](t x-n) e^{-x t} \mathrm{~d} t \\
& =\varphi\left(\frac{n}{x}\right) \int_{0}^{\infty} t^{n-1}\left[1-e^{-(p+1) t}\right](t x-n) e^{-x t} \mathrm{~d} t \\
& =\varphi\left(\frac{n}{x}\right)\left[x \int_{0}^{\infty} t^{n}\left[1-e^{-(p+1) t}\right] e^{-x t} \mathrm{~d} t-n \int_{0}^{\infty} t^{n-1}\left[1-e^{-(p+1) t}\right] e^{-x t} \mathrm{~d} t\right] \\
& =\varphi\left(\frac{n}{x}\right)\left[x \int_{0}^{\infty} t^{n} e^{-x t} \mathrm{~d} t-x \int_{0}^{\infty} t^{n} e^{-(x+p+1) t} \mathrm{~d} t-n \int_{0}^{\infty} t^{n-1} e^{-x t} \mathrm{~d} t+n \int_{0}^{\infty} t^{n-1} e^{-(x+p+1) t} \mathrm{~d} t\right] \\
& =\varphi\left(\frac{n}{x}\right)\left[x \frac{n !}{x^{n+1}}-x \frac{n !}{(x+p+1)^{n+1}}-n \frac{(n-1) !}{x^{n}}+n \frac{(n-1) !}{(x+p+1)^{n}}\right] \\
& =\varphi\left(\frac{n}{x}\right) n !\left[\frac{1}{x^{n}}-\frac{x}{(x+p+1)^{n+1}}-\frac{1}{x^{n}}+\frac{1}{(x+p+1)^{n}}\right] \\
& =\varphi\left(\frac{n}{x}\right) \frac{n !}{(x+p+1)^{n}}\left(1-\frac{x}{x+p+1}\right) \\
& =\varphi\left(\frac{n}{x}\right) \frac{n !(p+1)}{(x+p+1)^{n+1}} \\
& >0
\end{aligned}
$$

where we used the formula

$\frac{1}{x^{\omega}}=\frac{1}{\Gamma(\omega)} \int_{0}^{\infty} t^{\omega-1} e^{-x t} \mathrm{~d} t$

for real numbers $x>0$ and $\omega>0$, see $\left[1\right.$, p. 255, 6.1.1]. So we obtain that the function $\theta_{p, 1}(x)$ is completely monotonic on $(0, \infty)$.

Since

$(-1)^{n}[u(x) v(x)]^{(n)}=\sum_{i=0}^{n}\left(\begin{array}{c}n \\ i\end{array}\right)\left[(-1)^{i} u^{(i)}(x)\right]\left[(-1)^{n-i} v^{(n-i)}(x)\right]$,

the product of any two completely monotonic function is also completely monotonic on their common domain. On the other hand, the function $x^{\alpha-1}$ for $\alpha<1$ is clearly completely monotonic on $(0, \infty)$. Consequently the function $\theta_{p, \alpha}(x)=x^{\alpha-1} \theta_{p, 1}(x)$

for $\alpha \leq 1$ is completely monotonic on $(0, \infty)$.

Conversely, if $\theta_{p, \alpha}(x)$ is completely monotonic on $(0, \infty)$, then

$\frac{\mathrm{d} \theta_{p, \alpha}(x)}{\mathrm{d} x}=x^{\alpha-1}\left\{\alpha\left[\ln \frac{p x}{x+p+1}-\psi_{p}(x)\right]+\frac{p+1}{x+p+1}-x \psi_{p}^{\prime}(x)\right\} \leq 0$ 
for $x>0$, equivalently,

$\alpha \leq \frac{x \psi_{p}^{\prime}(x)-\frac{p+1}{x+p+1}}{\ln \frac{p x}{x+p+1}-\psi_{p}(x)}$

Employing L'Hôspital's rule and (1.6) results in

$\lim _{x \rightarrow \infty} \frac{x \psi_{p}^{\prime}(x)-\frac{p+1}{x+p+1}}{\ln \frac{p x}{x+p+1}-\psi_{p}(x)}=\lim _{x \rightarrow \infty} \frac{x \psi_{p}^{\prime \prime}(x)+\psi_{p}^{\prime}(x)+\frac{p+1}{(x+p+1)^{2}}}{\frac{1}{x}-\frac{1}{x+p+1}-\psi_{p}^{\prime}(x)}=\lim _{x \rightarrow \infty} \frac{\frac{p+1}{(x+p+1)^{2}}-x \sum_{k=0}^{p} \frac{2}{(x+k)^{3}}+\sum_{k=0}^{p} \frac{1}{(x+k)^{2}}}{\frac{1}{x}-\frac{1}{x+p+1}-\sum_{k=0}^{p} \frac{1}{(x+k)^{2}}}=1$,

so it is necessary that $\alpha \leq 1$. The proof is complete.

Second Proof. From (2.2) and by integration by part lead to

$$
\begin{aligned}
\theta_{p, 1}(x) & =-\int_{0}^{\infty}\left[1-e^{-(p+1) t}\right] \varphi(t) \frac{\mathrm{d} e^{-x t}}{\mathrm{~d} t} \mathrm{~d} t \\
& =\int_{0}^{\infty}\left\{\left[1-e^{-(p+1) t}\right] \varphi(t)\right\}^{\prime} e^{-x t} \mathrm{~d} t-\left.\left\{\left[1-e^{-(p+1) t}\right] \varphi(t) e^{-x t}\right\}\right|_{t=0} ^{t=\infty} \\
& =\int_{0}^{\infty}\left\{\left[1-e^{-(p+1) t}\right] \varphi^{\prime}(t)+(p+1) e^{-(p+1) t} \varphi(t)\right\} e^{-x t} \mathrm{~d} t .
\end{aligned}
$$

Therefore, for showing that the function $\theta_{p, 1}(x)$ is completely monotonic on $(0, \infty)$ for all $p \in \mathbb{N}$, it suffices to prove that the function

$\left[1-e^{-(p+1) t}\right] \varphi^{\prime}(t)+(p+1) e^{-(p+1) t} \varphi(t)$

is positive. Since the function $\varphi(t)$ is increasing on $(0, \infty)$, the derivative $\varphi^{\prime}(t)$ is positive on $(0, \infty)$. Further considering the limits in (2.4), the positivity of $\varphi(t)$ follows. As a result, the function (2.6) is positive.

The rest of the proof is the same as the first proof.

Remark 2.1. This paper is a slightly modified version of the preprint [9].

\section{Acknowledgements}

The second author was partially supported by the National Natural Science Foundation of China under Grant No. 11361038 and by the Foundation of the Research Program of Science and Technology at Universities of Inner Mongolia Autonomous Region under Grant No. NJZY14192, China.

\section{References}

[1] M. Abramowitz and I. A. Stegun (Eds), Handbook of Mathematical Functions with Formulas, Graphs, and Mathematical Tables, National Bureau of Standards, Applied Mathematics Series 55, 9th printing, Washington, 1970 .

[2] H. Alzer, On some inequalities for the gamma and psi functions, Math. Comp. 66 (1997), no. 217, 373-389; Available online at http://dx.doi.org/10.1090/S0025-5718-97-00807-7.

[3] T. M. Apostol, Introduction to Analytic Number Theory, Springer-Verlag, 1976.

[4] B.-N. Guo and F. Qi, Two new proofs of the complete monotonicity of a function involving the psi function, Bull. Korean Math. Soc. 47 (2010), no. 1, 103-111; Available online at http://dx.doi.org/10.4134/bkms.2010.47.1.103.

[5] B.-N. Guo, A.-Q. Liu, and F. Qi, Monotonicity and logarithmic convexity of three functions involving exponential function, J. Korea Soc. Math. Educ. Ser. B Pure Appl. Math. 15 (2008), no. 4, 387-392.

[6] B.-N. Guo and F. Qi, A simple proof of logarithmic convexity of extended mean values, Numer. Algorithms 52 (2009), no. 1, 89-92; Available online at http://dx.doi.org/10.1007/s11075-008-9259-7. 
[7] S. Guo and F. Qi, A class of completely monotonic functions related to the remainder of Binet's formula with applications, Tamsui Oxf. J. Math. Sci. 25 (2009), no. 1, 9-14.

[8] V. Krasniqi, T. Mansour, and A. Sh. Shabani, Some monotonicity properties and inequalities for $\Gamma$ and $\zeta$ functions, Math. Commun. 15 (2010), no. 2, 365-376.

[9] V. Krasniqi and F. Qi, Complete monotonicity of a function involving the p-psi function and alternative proofs, available online at http://arxiv.org/abs/1105.4928.

[10] V. Krasniqi and A. Sh. Shabani, Convexity properties and inequalities for a generalized gamma function, Appl. Math. E-Notes 10 (2010), 27-35.

[11] A.-Q. Liu, G.-F. Li, B.-N. Guo, and F. Qi, Monotonicity and logarithmic concavity of two functions involving exponential function, Internat. J. Math. Ed. Sci. Tech. 39 (2008), no. 5, 686-691; Available online at http://dx.doi.org/10.1080/00207390801986841.

[12] D. S. Mitrinović, J. E. Pečarić, and A. M. Fink, Classical and New Inequalities in Analysis, Kluwer Academic Publishers, Dordrecht-Boston-London, 1993.

[13] F. Qi, Bounds for the ratio of two gamma functions, J. Inequal. Appl. 2010 (2010), Article ID 493058, 84 pages; Available online at http://dx.doi.org/10.1155/2010/493058.

[14] F. Qi, P. Cerone, S. S. Dragomir, and H. M. Srivastava, Alternative proofs for monotonic and logarithmically convex properties of one-parameter mean values, Appl. Math. Comput. 208 (2009), no. 1, 129-133; Available online at http://dx.doi.org/10.1016/j.amc.2008.11.023.

[15] F. Qi and B.-N. Guo, Some properties of extended remainder of Binet's first formula for logarithm of gamma function, Math. Slovaca 60 (2010), no. 4, 461-470; Available online at http://dx.doi.org/10.2478/s12175-010-0025-7.

[16] R. L. Schilling, R. Song, and Z. Vondraček, Bernstein Functions, de Gruyter Studies in Mathematics 37, De Gruyter, Berlin, Germany, 2010.

[17] D. V. Widder, The Laplace Transform, Princeton University Press, Princeton, 1946.

[18] S.-Q. Zhang, B.-N. Guo, and F. Qi, A concise proof for properties of three functions involving the exponential function, Appl. Math. E-Notes 9 (2009), 177-183. 